\title{
North European species of Ceriporiopsis (Basidiomycota) and their Asian relatives
}

\author{
JUHA KINNUNEN and TUOMO NIEMELÄ
}

\begin{abstract}
Kinnunen, J. \& Niemelä, T. 2005: North European species of Ceriporiopsis (Basidiomycota) and their Asian relatives. - Karstenia 45: 81-90. ISSN 0453-3402.

The polypore genus Ceriporiopsis is revised, and a new combination is proposed: $\mathrm{Ce}$ riporiopsis pseudogilvescens (Pilát) Niemelä \& Kinnunen. It is the correct name for the species commonly known as $C$. resinascens (Romell) Domański; the latter is a northerly taxon with narrower spores and less resinous basidiocarp. Also the related $C$. aneirina (Sommerf.) Domański, C. balaenae Niemelä, C. gilvescens (Bres.) Domański, C. albonigrescens Núñez, Parmasto \& Ryvarden and C. cremea (Parmasto) Ryvarden are discussed and compared. The results are supplemented with an extensive list of spore size measurements and by a key to the species.
\end{abstract}

Key words: Basidiomycota, Ceriporiopsis albonigrescens, Ceriporiopsis aneirina, Ceriporiopsis balaenae, Ceriporiopsis cremea, Ceriporiopsis pseudogilvescens, Ceriporiopsis resinascens, polypore, taxonomy.

Juha Kinnunen and Tuomo Niemelä, Finnish Museum of Natural History, Botanical Museum, P.O. Box 7, FI-00014 University of Helsinki, Finland

\section{Introduction}

The genus Ceriporiopsis Donk is an assemblage of white-rot-causing basidiomycetes with monomitic structure and clamped hyphae. $\mathrm{Ce}$ riporiopsis as currently understood is obviously polyphyletic, as already noted by Ryvarden (1991; Ryvarden \& Gilbertson 1993). The use of molecular and other new methods has revealed that many of the species included in the genus actually belong to other genera: C. pannocincta (Boidin 1998, Koker et al. 2003), C. rivulosa (Boidin 1998, Kim et al. 2003, Koker et al. 2003), C. subvermispora (Greslebin et al. 2004), C. mucida (Rajchenberg 2003) and C. subrufa represent examples of this kind of aliens. In the future most likely still other taxa, now addressed to Ceriporiopsis, will find their place elsewhere, leaving behind a small genus of brownish polypores living on hardwood. Yao et al. (1999) found that $C$. gilvescens, the type species of the genus, is closely related to Aurantioporus alborubes- cens. According to Kim et al. (2003) Ceriporiopsis resinascens var. pseudogilvescens groups together with Antrodiella americana.

In this paper we summarize the knowledge of the genus from the North European point of view. Here the genus is treated sensu stricto, comprising species close to the generic type $C$. gilvescens. Ceriporiopsis subrufa is considered to belong elsewhere because of its strongly cyanophilous skeletal hyphae, a character alien to Ceriporiopsis.

\section{Materials and methods}

The specimens listed are deposited in the Botanical Museum of the University of Helsinki (H), unless otherwise indicated. Herbarium abbreviations are according to Holmgren et al. (1990). In sections Specimens examined, the following abbreviations are used: $\mathrm{NR}=$ Nature Reserve, $\mathrm{SNR}=$ Strict Nature Reserve, $\mathrm{NP}=$ National Park, $\mathrm{VF}=$ Virgin Forest. 
Microscopic studies were done and spores were measured from sections mounted in Cotton Blue (abbreviated CB): $0.1 \mathrm{mg}$ aniline blue (Merck 1275) dissolved in 60 g pure lactic acid; $\mathrm{CB}+$ means cyanophily, $\mathrm{CB}(+)$ weak but distinct cyanophilous reaction, $\mathrm{CB}-$ acyanophily. Amyloid and dextrinoid reactions were tested in Melzer's reagent (IKI): $1.5 \mathrm{~g}$ KI (potassium iodide), $0.5 \mathrm{~g} \mathrm{I}$ (crystalline iodine), $22 \mathrm{~g}$ chloral hydrate, aq. dest. $20 \mathrm{ml}$; IKI- means neither amyloid nor dextrinoid reaction.

As a rule 30 spores were measured from each specimen selected for a closer scrutiny. Measurements were done using $\times 1000$ magnification, phase contrast and oil immersion; eyepiece scale bar showed a $1-\mu \mathrm{m}$-grid, and dimensions were estimated subjectively with an accuracy of $0.1 \mu \mathrm{m}$. In presenting the variation of spore size, $5 \%$ of the measurements out of each end of the range are given in parentheses. $\mathrm{L}=$ mean length (arithmetical mean of all spores), $\mathrm{W}=$ mean width, $\mathrm{Q}=$ extreme values of the length/width ratios among the studied specimens, and $\mathrm{n}=$ the number of spores measured from given number of specimens.

The main reference books used were: Bondartsev (1953), Gilbertson \& Ryvarden (1986-1987), Ryvarden \& Gilbertson (1993-1994), Núñez \& Ryvarden (2001) and Hansen \& Knudsen (1997).

\section{Key}

A revised key to Ceriporiopsis in Northern Europe (including C. cremea and C. albonigrescens) is presented here.

1 Pores large (1-2 per mm), basidiospores 5.9-7.0 × 3.7-4.4 $\mu \mathrm{m}$ C. aneirina

- Pores small (3-6 per mm), basidiospores 3.0-6.0 × 1.7-3.5 $\mu \mathrm{m}$ 2

2 Basidiospores $1.7-2.3 \mu \mathrm{m}$ wide 3

Basidiospores wider than $2.3 \mu \mathrm{m}$

3 Basidiospores 1.7-2.0 $\mu \mathrm{m}$ wide; basidiocarp turning black when touched C. albonigrescens Basidiospores 1.8-2.3 $\mu \mathrm{m}$ wide C. gilvescens

4 Basidiospores 2.3-2.6 $\mu \mathrm{m}$ wide C. resinascens

- Basidiospores 2.6-3.5 $\mu \mathrm{m}$ wide C. balaenae

5 Basidiospores 3.6-4.2 $\mu \mathrm{m}$ long

- Basidiospores 4.5-6.0 $\mu \mathrm{m}$ long

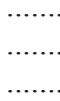
5

6 Basidiospores 4.5-6.0 $\mu \mathrm{m}$ long; oil globules common .. 6

- Basidiospores 4.5-6.0 $\mu \mathrm{m}$ long; no oil globules C. pseudogilvescens C. cremea

Keys for Ceriporiopsis s.l. have been published from tropical America by Loguercio-Leite et al. (2001), for Southern Europe by Pieri and Rivoire (1996), Central Europe by Domański (1972), all of Europe by Ryvarden and Gilbertson (1993) and Bernicchia and Ryvarden (2003). The Chi- nese species were keyed out by Wei and Dai (2004), and the whole East Asia is covered in the treatment by Núñez et al. (2001).

Spore dimensions of the treated species are summarized in Table 1. Measurements from the studied specimens are listed in Appendix 1.

Table 1. Spore dimensions of six Ceriporiopsis species.

\begin{tabular}{lllll}
\hline & $\mathrm{L} \times \mathrm{W}$ & $\mathrm{L}^{*}$ & $\mathrm{~W}^{*}$ & $\mathrm{Q}^{*}$ \\
\hline C. gilvescens & $3.6-4.6 \times 1.8-2.4$ & $3.7-4.2$ & $1.9-2.4$ & $1.80-2.20$ \\
C. balaenae & $3.6-4.2 \times 2.6-3.2$ & $3.8-4.2$ & $2.8-3.1$ & $1.30-1.50$ \\
C. pseudogilvescens & $4.0-6.0 \times 2.6-3.3$ & $4.5-5.5$ & $2.8-3.1$ & $1.50-1.80$ \\
C. resinascens & $4.0-6.0 \times 2.2-2.7$ & $4.5-5.5$ & $2.3-2.6$ & $1.80-2.20$ \\
C. cremea & $4.0-6.0 \times 2.7-3.5$ & $4.5-5.2$ & $2.8-3.1$ & $1.50-1.70$ \\
C. aneirina & $5.9-7.0 \times 3.7-4.4$ & $6.4-7.2$ & $3.6-4.4$ & $1.50-1.80$ \\
\hline
\end{tabular}




\section{Taxonomy}

Ceriporiopsis pseudogilvescens (Pilát) Niemelä \& Kinnunen, comb. nova - Figs. 1, 2.

Basionym: Poria pseudogilvescens Pilát, Bull. Soc. Mycol. France 51:378, 1936. - Type: Russia. Siberia: Tomsk Region, Vasyuganje, Betula pendula, X.1934 Krawtzew W 14 (PRM 498233, studied).
Leptoporus micantiformis Pilát, Bull. Soc. Mycol. France 51:358, 1936. - Type: Russia. Siberia: Tomsk Region, Vasyuganje, Chashanka River, Populus, 15.VIII.1934 Krawtzew W 4 (PRM 38285, studied).

Poria subpudorina Pilát, Sborn. Národ. Mus. Praze, B, Př́r. Vědy 9:105, 1953. - Type: Ukraine. Zakarpats'ka Region: Kosovská Polána, Jalinka, Salix caprea, VII.1930 Pilát (PRM 498304, studied).
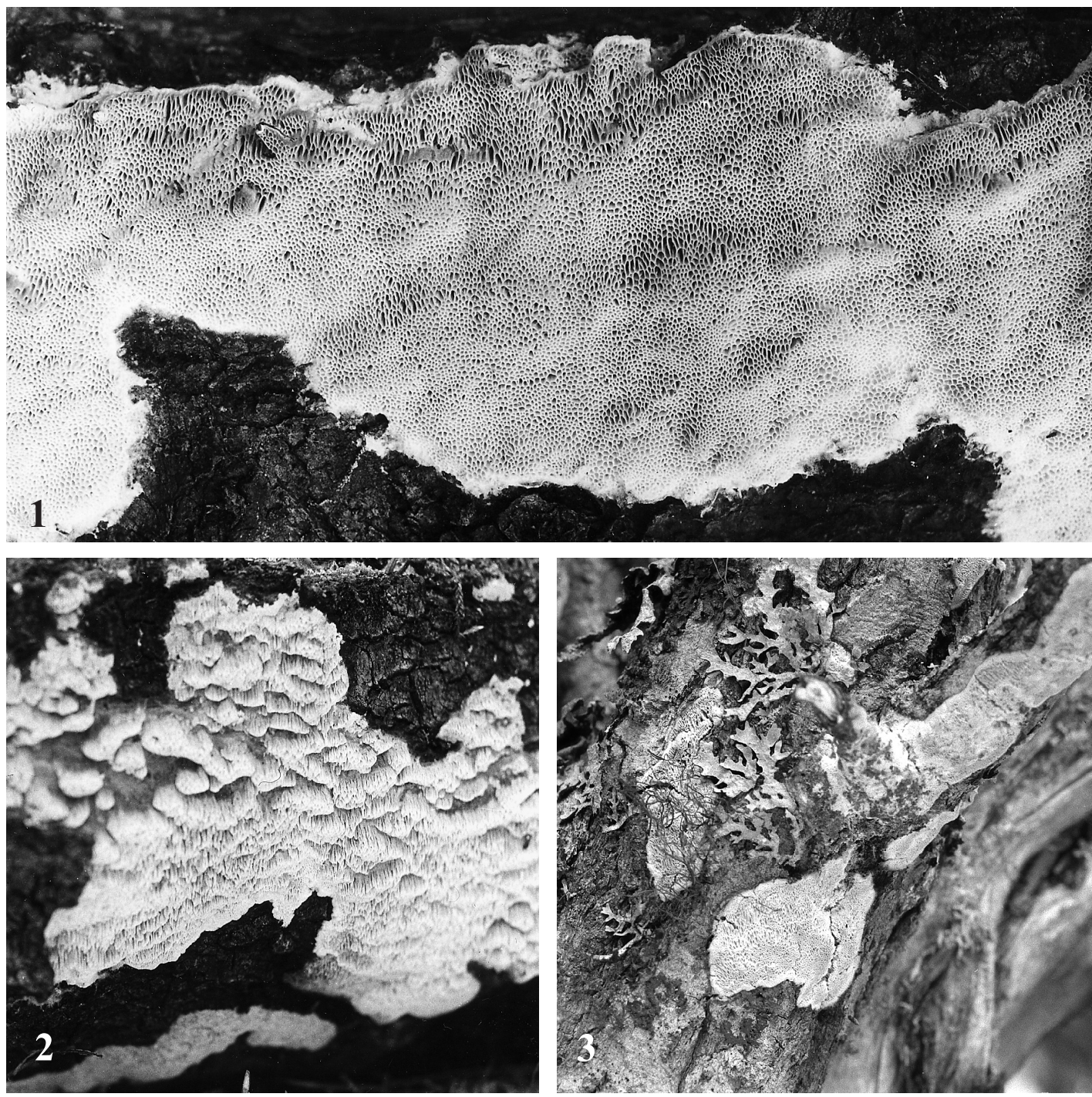

Figs. 1-3. Ceriporiopsis species, photographed in situ. - 1: Ceriporiopsis pseudogilvescens, young resupinate basidiocarp. Specimen Niemelä 2775, $\times 1.8$. 2: C. pseudogilvescens, pseudopilei. Niemelä 2765, $\times 0.9$. - 3: C. resinascens, basidiocarps at the base of dead branch, still attached to moribund Salix caprea. Niemelä 2051, ×0.9. 
$=$ Ceriporiopsis resinascens auct., non sensu typi.

Basidiocarp annual, resupinate, small or up to 15 $\mathrm{cm}$ wide, soft and easily breakable when young, hard and brittle when dry; sometimes developing small, nodulose pseudopilei. Sterile margin sordid straw-coloured, paler than the tubes, 1-3 $\mathrm{mm}$ wide, not rhizomorphic. Pore surface at first white, during the best growth rose-tinted, later and when dry resin brown; pore orifices covered with light pruina. Pores angular, variable, in young margin 2-3(-4) per mm; when growing on a sloping log-side pores larger, sinuous. Section: subiculum cream coloured, tubes resin brown, contrast clear; thickness $2-3 \mathrm{~mm}$.

Monomitic, hyphae with clamp connections, in subiculum both thin- and thick-walled (appearing dimitic), IKI-, $\mathrm{CB}(+)$, thick-walled hyphae sometimes amyloid. Subiculum hyphae $2-3.5(-4) \mu \mathrm{m}$ in diameter and sometimes with scattered crystals, among the thin-walled hyphae also some thick-walled hyphae present, with a narrow cavity, clamps and branches; hyphae interwoven. Dissepiment hyphae thin-walled, 2-2.9 $\mu \mathrm{m}$ in diameter, longitudinally interwoven; embedded in resinous matter (especially in subhymenium). Basidia 13-22 × 4.5-5.5(-6) $\mu \mathrm{m}$, basidioles $16-22 \times 4-6 \mu \mathrm{m}$, narrowly claviform, forming a tight palisade. Spores broadly ellipsoid, thin-walled, IKI-, CB-, (3.4-)3.8-6.0 $(-7.6) \times(2.4-) 2.6-3.1(-3.6) \mu \mathrm{m}, \mathrm{L}=4.64 \mu \mathrm{m}$, $\mathrm{W}=2.89 \mu \mathrm{m}, \mathrm{Q}=1.50-1.80(\mathrm{n}=780 / 26)$.

The name $C$. resinascens has been interpreted in many ways in the past. Domański (1972) described three varieties and one form. Pilát's broad-spored species Poria pseudogilvescens has usually been considered to be a synonym or variety of $C$. resinascens. However, the type of $C$. resinascens has in average narrower spores (as already stated by Romell in the protologue; see Tabs. 2-3), indicating a different taxon. Ryvarden (1978) measured the spores of the type to be $6-8.5 \times 2.5-3 \mu \mathrm{m}$. Variation in spore dimensions can be great between Ceriporiopsis specimens (Tabs. 1-2), and in a single specimen many kinds of spores can be found. Usually spore length is more variable, while the width stays more or less constant. Hence we consider that two taxa are involved, and pseudogilvescens is the earliest name to the taxon with broader spores.
As stated by Egeland (1911) and also suspected by Bourdot and Galzin (1928), Karsten never published a taxon with the name Poria starbaeckii which might have been an earlier name for this taxon (Lowe 1956).

Ceriporiopsis pseudogilvescens is widespread from Europe to Siberia.

Specimens examined: Finland. Uusimaa: Sipoo, Hindsby, Salix, 8.VII.1987 Saarenoksa 11487; Myras-Hindsby, Populus, 15.IX.1991 Saarenoksa 34391. Vantaa, Västra, Betula, 15.IX.1995 Niemelä 5904. Helsinki, KumpulaToukola, Salix, 28.VIII.1979 Niemelä 1681; Vanhakaupunki, Annala, Salix, 14.VII.1996 Saarenoksa 04496; Viikki, Salix, 21.IX.1996 Saarenoksa 10096. Tammisaari, Tvärminne, Corylus, 27.VIII.1971 Niemelä \& Väisälä. Mäntsälä, Hirvihaara, Populus, 24.VI.1987 Niemelä 4011. Etelä-Häme: Lammi, Evo, Kotinen VF, Populus, 10.IX.1982 Niemelä 2775. Etelä-Savo: Juva, Halkokangas, Salix, 15.VII.1996 Vehmaa 852. Pohjois-Savo: Kangaslampi, Joutenniemi, Salix, 5.X.1991 Haikonen 13253. Pohjois-Karjala: Lieksa, Koli, Populus, 9.VIII.1979 Kotiranta 1469, Niemelä 1659, 1662; Patvinsuo NP, Populus, 14.IX.1989 Penttilä 1245b. Ilomantsi, Mekrijärvi, Populus, 26.VIII.1996 Jakobsson 1938b. Kainuu: Hyrynsalmi, Tulisuo-Varpusuo, Populus, 16.IX.1990 Penttilä 1772b. Kuhmo, Teerisuo-Losonsuo, Populus, 4.IX.1990 Penttilä 1646. Perä-Pohjanmaa: Tervola, Pisavaara SNR, Liljalaki, Salix, 24.VIII.1980 Niemelä 2051, Kotiranta 2143; Populus, 25.VIII.1980 Kotiranta 2163 \& Niemelä. Sweden. Västmanland: Sala, Skuggan, Fiskarbo, 9.VIII.1949 Morander 1435 (UPS). Russia. Leningrad Region: Veps Forest, Populus, 2.VI.2000 Manninen 786. Tosno Dist.: Lisino Forest, Salix, 7.IX.1960 Bondartseva 277 (LE 25200). Siberia: Tomsk Region (see types). Kemerovo Region: Promyshlennov. papermill, Populus, IX.1966 Shukov (LE 25193). Belarus. Belovezha VF, Pezezovo forestry, $S a$ lix, 27.VIII.1966 Bondartseva 229 (LE 27623). Ukraine. Zakarpats'ka Region (see types). Czech Republic. Bohemia: Sobeslav W, Salix, 25.VII.1973 Niemelä, Kotlaba \& Pouzar. France. Savoie: Méribel-les-Allues, angiosperm, 1.IX.1990 Pieri. Canada. Québec: Poste-de-la-Baleine, Salix, 31.VII.1982 Niemelä 2597.

\section{Ceriporiopsis resinascens (Romell) Domański} - Fig. 3

Polyporus resinascens Romell, Ark. Bot. 11 (3): 20, 1912. - Lectotype: Sweden. Torne Lappmark: Jukkasjärvi, Jebrenjokk, 28.VIII.1910 Romell 43 (S F14660).

Tyromyces polyetes Parmasto, Bot. Mater. Otd. Spor. Rast. Bot. Inst. Akad. Nauk SSSR 12:239, 1959. - Type: Estonia. Harjumaa: Keila, Haju, Populus, VII.1951 Parmasto (LE 23049, studied).

Basidiocarp annual, resupinate, small, soft and easily breakable, stiff when dry. Margin creamy 
white, paler than the pore surface. Pore surface first cream coloured, later and when dry straw coloured or olive brown, when old greyish ochraceous brown, rusty colours present only rarely; pores at young margin 3-5(-6) per mm, growing larger with age. Section: subiculum cream coloured; tubes concolorous with pore surface; thickness 1-2 mm.

Monomitic, hyphae with clamp connections, hyphae $\mathrm{CB}(+)$, IKI-. When young context hyphae thin-walled, when old both thin- and thickwalled with occasional clamps (then appearing dimitic); hyphae (1.7-)2.2-3 $\mu \mathrm{m}$, interwoven, crystals sometimes present in between the hyphae; basal layer sometimes differentiated, with thin-walled hyphae, 3.5-5.3 $\mu \mathrm{m}$. Dissepiment hyphae 2-2.7(-3) $\mu \mathrm{m}$, thin-walled, longitudinally winding, a few yellow oil droplets seen between the hyphae. Basidia 14-19(-21) × 4.5-5.5 $\mu \mathrm{m}$. Spores narrower than in $C$. pseudogilvescens or C. balaenae, cylindrical or tapering only a little, thin-walled, IKI-, CB-, (4.5-)4.9-6.2(-6.5) $\times$ (2.1-)2.2-2.6(-2.9) $\mu \mathrm{m}, \mathrm{L}=5.0 \mu \mathrm{m}, \mathrm{W}=2.4 \mu \mathrm{m}$, $\mathrm{Q}=1.80-2.20(\mathrm{n}=660 / 22)$.

According to the spore dimensions, the specimens of Komarova (1959) and Christiansen (1960) represent this species. It was colour illustrated by Niemelä (2005).

Specimens examined: Finland. Varsinais-Suomi: Kaarina, Littoistenjärvi W, Populus, 20.IX.1936 Laurila. Uusimaa: Tammisaari, Bromarv, Framnäs, Populus, 4.X.1975 Niemelä 532. Helsinki, Viikki, Salix, 16.VI.1985 Saarenoksa 05185; Mustavuori, Populus, 27.VII.1984 Saarenoksa 23784; Salix, 24.VIII.1994 Saarenoksa 09894. Tuusula, Lahela, Malus, 8.IX.1991 Saarenoksa 28391; Salix, 13.VIII.1992 Saarenoksa 11292. Sipoo, Myras-Hindsby, Salix, 24.VII.1990 Saarenoksa 11790. Etelä-Häme: Ylöjärvi, Lempiäniemi, Populus, 22.VIII.1993 Söderholm 2122. Lammi, Evo, Kotinen VF, Populus, 17.IX.1985 Niemelä 3281. Kainuu: Puolanka, Kuirivaara, Muroselkä, Populus, 24.VIII.1993 Penttilä 4453. Oulun Pohjanmaa: Oulu, Letonniemi, sine matrice, 4.VIII.2003 Halonen, Pippola \& Wannas (OULU, H). Koillismaa: Salla, Värriö SNR, Sorbus, 26.VIII.1987 Renvall 77 \& Renvall; 2.VIII.1988 Renvall 986 \& Renvall; Populus, 23.IX.1988 Renvall 1387 \& Renvall. Kittilän Lappi: Aakenus, Vasalaki, Populus, 29.VIII.2000 Niemelä 6823. Sweden. Torne Lappmark (see types). Estonia. Harjumaa: Keila (see types). Vääna, Vääna-Jõesuu, Salix, 16.IX.1993 Kinnunen 1. Raplamaa: Kohila, Salix, 20.VIII.1942 Jakobsen (TAA 158149). Sine loco, Alnus, 19.VIII.1991 Parmasto (TAA 126661). Russia. Karelian Rep.: Medvezhegorsk Dist., River Vichka, Populus, 23.VI.1942 Laurila 549. Czech Republic. Moravia: Vysoká, Populus, 22.VIII.1991 Vampola.

\section{Ceriporiopsis aneirina (Sommerf.: Fr.)} Domański

Polyporus aneirinus Sommerf., Fl. Lapponica suppl.: 278, 1826: Fr. 1828. - Type: Norway. Nordland: Saltdal, Saltdalen, in trunco subputr. Populi, X.1820 Sommerfelt (O F72702; K).

Antrodia serena P. Karst., Medd. Soc. F. Fl. Fenn. 6:10, 1881. - Type: Finland. Etelä-Häme: Tammela, Mustiala, Populus, 5.X.1880 Karsten 2505-2506 (H, BPI 242279, S F6866, studied).

Poria fulvescens Bres., Atti I.R. Accad. Roveret. Sci. III 3(3):81, 1897. - Type: Slovakia. Stiavnické Pohorie: Prenčov, Mt. Sitno, Populus tremula, 7.VII.1892 Kmet' (BPI 318507, S F7375).

Poria wasjuganica Pilát, Bull. Soc. Mycol. France 51:382, 1936. -Type: Russia. Siberia: Tomsk Region, Vasyuganje, Populus, 27.IX.1934 Krawtzew W 182 (PRM 181718, studied).

Basidiocarp annual, resupinate, small or middle-sized, quite soft, stiff when dry; at first seen as round or elliptic patches, later merging together. Margin white, thin, when dry paler than pores, $1-3 \mathrm{~mm}$. Pore surface at first straw white or young amber, then cream or honey coloured, when dry resin brown; pores angular, shallow, 1-2(-3) per mm, dissepiments split when old and then pores larger. Section: subiculum white, very thin; tubes resin coloured, contrast clear; thickness 1-2 mm. Young basidiocarp with an aromatic odour of honey, apple, or rind of grapefruit, becoming stronger 1-2 hours after collecting; old and dry with no smell; taste mild.

Monomitic, hyphae with clamp connections, generative hyphae thin-walled, IKI-, $\mathrm{CB}(+)$, in subiculum 3.8-5(-6) $\mu \mathrm{m}$ in diameter, with a large lumen, some hyphae covered with sandy crystals (sometimes numerous). Dissepiment hyphae thin-walled, (2.6-)2.8-4.2 $\mu \mathrm{m}$ in diameter, longitudinally interwoven. Oil droplets few or none. Basidia 20-27 × 6-7(-8) $\mu \mathrm{m}$; basidia and basidioles apically bulbous, making the hymenial palisade irregular. Spores ellipsoid, thinwalled, IKI-, CB-, (5.3-)5.6-7.0(-8.0) × (3.3-) 3.7-4.4(-4.6) $\mu \mathrm{m}, \mathrm{L}=6.27 \mu \mathrm{m}, \mathrm{W}=3.82 \mu \mathrm{m}$, $\mathrm{Q}=1.50-1.80(\mathrm{n}=390 / 13)$.

Specimens examined: Finland. Uusimaa: Pernaja, Fasarby, Populus, 10.I.1995 Särkkä 413b. Artjärvi, Ratula manor, Sorbus, 17.VIII.1990 Haikonen 11994. Helsinki, 
Populus, 4.XII.1859 Karsten 2507. Mäntsälä, Numminen, Populus, 10.X.1985 Haikonen 6620. Vähäjärvenkalliot, Populus, 20.V.1997 Bonn 207. Vantaa, Mustavuori, Populus, 7.IX.1986 Saarenoksa 37086. Satakunta: Kankaanpää, Venesjärvi, Populus, 10.VI.1935 Laurila 715. Etelä-Häme: Tammela (see types). Lahti, Populus, 1988 Niemelä 4545. Perä-Pohjanmaa: Tervola, Pisavaara SNR, Populus, 25.VIII.1980 Niemelä 2065, Kotiranta 2168. Estonia. Jõgevamaa: Puurmani, Alam-Pedja NR, Roka, Populus, 8.X.2001 Niemelä 7249, 7250. Russia. Bryansk Region: Trubchevsk Dist., Kokorevka, Populus, VI.1915 Bondartsev (LE 27108). Siberia: Tomsk Region (see types). Altay Rep., Ust'-Koksa Dist., Kajtanak, Populus, 4.IX.1968 Bondartseva (LE 27105). Novosibirsk Region: Togutsin, Derguvsk forestry, Populus, VIII.1965 Shukov (LE 27107). Krasnoyarsk Terr.: Stolby NR, Populus, 15.V.1926 Yavorskiy 730 (LE 27102).

\section{Ceriporiopsis balaenae Niemelä}

Naturaliste Canadien (Rév. Ecol. Syst.) 112:449, 1985. - Type: Canada. Québec: Poste-de-laBaleine, Salix, 12.VIII.1982 Niemelä 2752 (H, isotype DAOM).

Basidiocarp annual, resupinate, small, soft when fresh, fragile when dry. Margin thin, white. Pore surface straw-white, tubes honey yellow, dry tubes light amber coloured; when young bruised parts apricot coloured and translucent. Pores angular or slightly sinuous, when old orifices becoming lacerate, pores (1-)2-3 per mm. Section: subiculum cream coloured, only $0.5 \mathrm{~mm}$ thick, tubes oily or vaxy translucent, total thickness $2-5 \mathrm{~mm}$. No distinct odour, taste mild.

Monomitic, generative hyphae with clamp connections, thin-walled, IKI-, CB-, 2.8-3.5 $\mu \mathrm{m}$ in diameter. Subiculum hyphae loosely interwoven; no encrustations; dissepiment hyphae interwoven, IKI grey (reaction slow). Resinous matter sometimes present on subiculum hyphae. Basidia clavate, $14-18 \times 4.5-5 \mu \mathrm{m}$. Spores ellipsoid or ovoid, thin-walled, IKI-, CB- (contents faintly $\mathrm{CB}(+),(3.4-) 3.6-4.6(-5.0) \times(2.5-) 2.7-$ $3.3(-3.5) \mu \mathrm{m}, \mathrm{L}=4.03 \mu \mathrm{m}, \mathrm{W}=2.89 \mu \mathrm{m}, \mathrm{Q}=1.30$ $1.52(\mathrm{n}=480 / 16)$.

The appearance of this species varies a lot, as Vampola and Pouzar (1996) have noticed. The ecology is characteristic: the species is usually found on Salix in riverside thickets or other moist sites. The species must be commoner than what the few collections so far indicate.
Specimens examined: Finland. Uusimaa: Helsinki, Vanhakaupunki, Alnus, 17.IX.1994 Saarenoksa 18894, 8.X.1994 Saarenoksa 27794 \& Kotiranta; Salix, 15.VIII.1996 Saarenoksa 08096; Mustavuori, Salix, 4.X.1995 Saarenoksa 16395, 16495. Pohjois-Savo: Leppävirta, Sarkamäki, Salix, 2.VII.1993 Haikonen 15315. Perä-Pohjanmaa: Kemi, Perta-Aapa, Salix, 12.II.1991 \& 15.II.1991 Kamula. Inarin Lappi: Utsjoki, Kevo, Salix, 5.IX.1970 Niemelä 408b; 16.VIII.1987 Kotiranta 6371; 15.VIII.1995 Heinonen \& Heinonen 30895F. Sweden. Uppland: Ohersta Storsjön, Salix, 19.II.1994 Toresson. Värmdö, Salix, 4.XII.1994 Toresson. Canada. Québec: Poste-de-la-Baleine, Salix, 12.VIII.1982 Niemelä 2750, 2752 (type), 2753

\section{Related species}

\section{Ceriporiopsis gilvescens (Bres.) Domański}

Poria gilvescens Bres., Annales Mycol. 6:40, 1908. - Type: France. Vosges, Fagus, s.d., Bourdot 3666 (BPI 240221), 3810 (NY).

Tyromyces allantoideus M.P. Christiansen, Dansk Bot. Ark. 19:364, 1960. Type: Denmark. Falster: Fagus, XII.1929 F.H. Møller (C, studied).

Basidiocarp annual, resupinate, effused; when fresh waxy and soft, when dry hard and fragile; margin white cream with pinkish or reddish tint, pore surface when fresh at first whitish, when dry ochraceous. Pores angular, lacerate, 4-5 per mm. Taste mild.

Monomitic, hyphae thin-walled, with clamp connections, IKI-, CB-, in trama $2-5 \mu \mathrm{m}$ in diameter. Subiculum hyphae tightly packed, parallel, yellowish. Dissepiment hyphae subparallel, thin-walled, often slightly encrusted, 2.5-3 $\mu \mathrm{m}$. Dissepiment edges with rod-shaped crystals. No cystidia. Basidia clavate, $15-18 \times 4-5.5 \mu \mathrm{m}$. Spores cylindrical, hyaline, smooth, IKI-, $\mathrm{CB}(+)$, $(3.5-) 3.6-4.4(-4.6) \times(1.6-) 1.8-2.4(-2.5) \mu \mathrm{m}$, $\mathrm{L}=3.99 \mu \mathrm{m}, \mathrm{W}=2.05 \mu \mathrm{m}, \mathrm{Q}=1.95(\mathrm{n}=90 / 3)$.

The type species of the genus. In northern Europe the species is found in South Sweden and Denmark.

Specimens examined: Denmark. Falster (see types). Czech Republic. Moravia: Hostýnské Vrchy, Černava, Fagus, 9.VIII.1973 Niemelä. Slovakia. Kremnické Pohorie: Badinský prales, Fagus, 4.VIII.1973 Niemelä, Kotlaba \& Pouzar. China. Jilin Prov.: Wangqing, Populus, 27.V.1993 Dai 246. 


\section{Ceriporiopsis albonigrescens Núñez, Parmasto \& Ryvarden}

Fungal Diversity 6:107, 2001. - Type: Russia. Primorye Terr., Ternei Dist., Sikhote-Alin Biosphere Reserve, Maisa, Abies nephrolepis, 11.IX.1979 Parmasto (TAA 52514, O, H, studied).

Basidiocarp annual, resupinate, adnate, widely effused (up to $15 \mathrm{~cm}$ ), soft when fresh, soft and fragile when dry, up to $3 \mathrm{~mm}$ thick. Margin 1-2 mm wide, white, cottony, no rhizomorphs. Subiculum very thin (usually less than $0.1 \mathrm{~mm}$ ), white, cottony. Tubes 1-3.5 mm long, concolorous with pore surface, dissepiments thin. Pore surface creamy white when fresh, cream coloured when dry, blackish brown when touched. Pores angular, 4-5 per mm.

Monomitic, hyphae thin-walled, with clamp connections, IKI-, CB-, in trama $2.5-3.5 \mu \mathrm{m}$ in diameter, densely packed in a parallel fashion. Hyphae in context 3-6 $\mu \mathrm{m}$ in diameter, more loosely interwoven. Rhomboid crystals present between hyphae. Dissepiment edges with a few robust crystals. No cystidia. Basidia clavate, 13-15 $\times 4-5 \mu \mathrm{m}$. Spores short-cylindric to oblong-ellipsoid, one side flattened, thin-walled, smooth, hyaline, IKI-, CB-, (2.9-)3.0-3.4(-3.7) $\times(1.6-) 1.7-2.0 \mu \mathrm{m}, \mathrm{L}=3.19, \mathrm{~W}=1.79, \mathrm{Q}=1.78$ $(\mathrm{n}=30 / 1)$.

The rhomboid crystals in between hyphae, coniferous host, white pocket rot, small spores and blackening in bruised parts are characters that are otherwise alien to the genus Ceriporiopsis. Therefore it is likely that this species will later be moved to some another genus.

Specimen examined: Russia. Primorye Terr. (see type).

\section{Ceriporiopsis cremea (Parmasto) Ryvarden}

Fibuloporia cremea Parmasto, Issled. Prirody Dal'nego Vostoka: 255, 1963. - Type: Russia. Kamchatka: Klyuchi, Populus, 18.VIII.1960 Parmasto (TAA 13599, studied).

Basidiocarp annual, resupinate, effused up to $10 \mathrm{~cm}$, soft when fresh, fragile when dry, margin white and rhizomorphic; pore surface pale cream. Pores $2-3$ per mm, angular, dentate.

Monomitic, generative hyphae thin-walled, with clamp connections, IKI-, CB-; hyphae spaced in upper subiculum next to wood, 2-3.5 $\mu \mathrm{m}$, lower subiculum tightly packed, 3.2-5.2 $\mu \mathrm{m}$, dissepiment hyphae subparallel, 2.4-3.6 $\mu \mathrm{m}$. Subiculum hyphae covered with coarse to tiny encrustations, crystals seen also among dissepiment hyphae. No cystidia, cystidioles common. Basidia clavate, 12-20 × 5-6 $\mu \mathrm{m}$. Spores subcylindrical, thin-walled, hyaline, smooth, IKI-, CB-, (3.7-)3.9-6.4(-6.9) × (2.7-)2.9-3.5 $(-3.8) \mu \mathrm{m}, \mathrm{L}=4.65 \mu \mathrm{m}, \mathrm{W}=2.99 \mu \mathrm{m}, \mathrm{Q}=1.56$ $(n=120 / 4)$.

Specimens examined: Russia. Sakha Rep.: Oimyakon, Burustakh, Andygytshan, Salix (Chosenia) arbutifolia, 30.VIII.1972 Parmasto (TAA 55919). Primorye Terr.: Hasan, Kedrovaya Pad', Acer mandschurica, 22.VII.1985 Parmasto (TAA 106200). Ternei Dist.: Sikhote-Alin Biosphere Reserve, Maisa, Populus maximoviczii, 16.IX.1990 Parmasto (TAA 151113). Kamchatka: Klyuchi (see type). Elizovo Region, Vakhtolka, Salix sachalinensis, 22.IX.1960 Parmasto (TAA 12481). Kozyrevsk, Populus, 24.VIII.1960 Parmasto (LE 22424, LE 206290).

Acknowledgements: The Ministry of the Environment (Finland) is thanked for a research grant (YM 175/5512/2004). Many thanks to Reima Saarenoksa, who drew our attention to the difference between $C$. resinascens and C. pseudogilvescens. The curators of the herbaria C, LE, O, PRM, S, TAA and UPS are thanked for providing specimens. Alexander Kovalenko and Margarita Bondartseva are thanked for their hospitality during a visit in St.Petersburg.

\section{References}

Bernicchia, A. \& Ryvarden, L. 2003: A new white-rot polypore from Italy. - Mycotaxon 88: 219-224.

Boidin, J. 1998: Taxonomie moleculaire des Aphyllophorales. - Mycotaxon 66: 445-491.

Bondartsev, A. 1953: Trutovye griby evropeyskoy chasti SSSR i Kavkaza. - Akad. Nauk SSSR, Moskva \& Leningrad. 1107 pp.

Bourdot, H. \& Galzin, A. 1928: Hyménomycètes de France. Hetérobasidiés-Homobasidiés gymnocarpes. - Soc. Mycol. France, Sceaux. 761 pp.

Christiansen, M.P. 1960: Danish resupinate fungi 2. Homobasidiomycetes. - Dansk Bot. Arkiv 19: 57-388.

Domański, S. 1972: Fungi, Polyporaceae 1, Mucronoporaceae 1. - Foreign Sci. Publ., Warsaw. 235 pp.

Egeland, J. 1911: Meddelelser om norske hymenomyceter 1. - Nyt Magazin for Naturvidenskaberne 51: 341-380.

Gilbertson, R.L. \& Ryvarden, L. 1986-1987: North American polypores 1-2. - Fungiflora, Oslo. 885 pp.

Greslebin, A., Nakasone, K.K. \& Rajchenberg, M. 2004: Rhizochaete, a new genus of phanerochaetoid fungi. - Mycologia 96: 260-271.

Hansen, L. \& Knudsen, H. (eds.) 1997: Nordic macromycetes 3. Heterobasidioid, aphyllophoroid and gastromycetoid Basidiomycetes. - Nordsvamp, Copenhagen. 444 pp. 
Holmgren, P.K., Holmgren, N.H. \& Barnett, L.C. (eds.) 1990: Index herbariorum 1. The herbaria of the world. 8th ed. - New York Bot. Garden, Bronx. 693 pp.

Kim, S.Y., Park, S.Y., Ko, K.S. \& Jung, H.S. 2003: Phylogenetic analysis of Antrodia and related taxa based on partial mitochondrial SSU rDNA sequences. - Antonie van Leeuwenhoek 83: 81-88.

Koker, T.H. de, Nakasone, K.K., Haarhof, J., Burdsall, H.H. \& Janse, B.J.H. 2003: Phylogenetic relationships of the genus Phanerochaete inferred from the internal transcribed spacer region. - Mycol. Res. 107: 1032-1040.

Komarova, E.P. 1959: Species rarae et formae novae Polyporacearum in Rossia alba inventae. - Bot. Mat. Otd. Spor. Rast. Bot. Inst. Akad. Nauk SSSR 12: 255.

Loguercio-Leite, C., Gonçalves, G.V. de Costa \& Ryvarden, L. 2001: Studies in neotropical polypores 13 . Ceriporiopsis cystidiata sp. nov. - Mycotaxon 79: 285-288.

Lowe, J.L. 1956: Type studies of the polypores described by Karsten. - Mycologia 48: 99-125.

Niemelä, T. 2005: Käävät, puiden sienet. Polypores, lignicolous fungi. - Norrlinia 13:1-320. [In Finnish, with English summary]

Núñez, M., Parmasto, E. \& Ryvarden, L. 2001: New and interesting polypores from East Russia. - Fungal Diversity 6: 107-114.
Núñez, M. \& Ryvarden, L. 2001: East Asian polypores 2. Polyporaceae sensu lato. - Synopsis Fungorum 14: 165-522.

Pieri, M. \& Rivoire, B. 1996: A propos de quelques polypores (Aphyllophoromycetideæ) rares ou critiques récoltes recemment 1. - Bull. Soc. Mycol. France 112: $163-187$.

Rajchenberg, M. 2003: Taxonomic studies on selected Austral polypores. - Australian Syst. Bot. 16: $473-$ 485.

Ryvarden, L. 1978: Polyporaceae of North Europe 2. - Fungiflora. Oslo. 286 pp.

Ryvarden, L. 1991: Genera of polypores. Nomenclature and taxonomy. - Synopsis Fungorum 5: 1-363.

Ryvarden, L. \& Gilbertson, R.L. 1993-1994: European polypores 1-2. - Fungiflora, Oslo. 743 pp.

Vampola, P. \& Pouzar, Z. 1996: Notes on some species of genera Ceriporia and Ceriporiopsis (Polyporaceae). - Czech Mycol. 48: 315-324.

Wei, Y.L. \& Dai, Y.C. 2004: Notes on Ceriporiopsis (Aphyllophorales) in China. - Fungal Science 19: 47-51.

Yao, Y.J., Pegler, D.N. \& Chase, M.W. 1999: Application of ITS (nrDNA) sequences in the phylogenetic study of Tyromyces s.1. - Mycol. Res. 103: 219-229. 
Appendix 1. Spore measurements of Ceriporiopsis species. Of the specimens with less than 30 measured spores, only the averages are given.

\begin{tabular}{|c|c|c|c|c|c|}
\hline Specimen & $\mathrm{L} \times \mathrm{W}$ & $\mathrm{L}^{*}$ & $\mathrm{~W}^{*}$ & Q & $\mathrm{Q}^{*}$ \\
\hline \multicolumn{6}{|l|}{ C. gilvescens } \\
\hline Dai 246 & $3.9-4.3(-4.6) \times(1.6-) 1.8-2.1$ & 4.12 & 1.96 & $1.90-2.50$ & 2.10 \\
\hline 1929 Møller (T. allantoideus) & & 4.10 & 1.83 & & 2.25 \\
\hline 1973 Niemelä & $(3.5-) 3.6-4.1(-4.2) \times(1.7-) 1.8-2.1(-2.2)$ & 3.78 & 1.97 & $1.62-2.17$ & 1.91 \\
\hline 1973 Niemelä, Kotl. \& Pouz. & $(3.7-) 3.8-4.4(-4.5) \times 2.0-2.4(-2.5)$ & 4.08 & 2.21 & $1.68-2.10$ & 1.85 \\
\hline \multicolumn{6}{|l|}{ C. pseudogilvescens } \\
\hline Bondartseva 229 & $(4.3-) 5.1-6.7(-7.6) \times(2.7-) 2.8-3.2$ & 5.71 & 2.97 & $1.48-2.45$ & 1.92 \\
\hline Bondartseva 277 & $(3.6-) 3.8-4.2(-4.5) \times 2.6-3.0(-3.2)$ & 4.00 & 2.82 & $1.26-1.58$ & 1.42 \\
\hline Haikonen 13523 & $(3.9-) 4.0-4.7 \quad \times(2.7-) 2.8-3.2(-3.3)$ & 4.28 & 2.93 & $1.31-1.62$ & 1.46 \\
\hline Jakobsson $1938 b$ & $(3.9-) 4.2-5.0(-5.2) \times 2.7-3.2$ & 4.60 & 2.92 & $1.33-1.81$ & 1.57 \\
\hline Kotiranta 1469 & $(3.9-) 4.3-5.3(-5.7) \times(2.4-) 2.6-3.0(-3.1)$ & 4.79 & 2.81 & $1.42-1.93$ & 1.70 \\
\hline Krawtzew W 4 (L. micantif.) & $4.2-5.1(-5.2) \times(2.7-) 2.8-3.2(-3.3)$ & 4.67 & 2.96 & $1.31-1.86$ & 1.58 \\
\hline Krawtzew W 14 (type) & $(4.1-) 4.2-5.0(-5.7) \times 2.6-3.0(-3.1)$ & 4.58 & 2.83 & $1.40-1.90$ & 1.62 \\
\hline Manninen 786 & $(3.5-) 3.8-4.5(-4.8) \times 2.5-3.1$ & 4.12 & 2.79 & $1.29-1.64$ & 1.48 \\
\hline Niemelä 1659 & $(4.3-) 4.6-6.0(-6.2) \times(2.6-) 2.8-3.2$ & 5.25 & 2.99 & $1.47-2.00$ & 1.76 \\
\hline Niemelä 1681 & $4.0-5.3(-5.5) \times 2.5-3.0$ & 4.60 & 2.76 & $1.33-2.00$ & 1.67 \\
\hline Niemelä 2597 & $(4.0-) 4.3-5.6(-6.0) \times 2.9-3.4(-3.6)$ & 4.95 & 3.10 & $1.38-1.90$ & 1.60 \\
\hline Niemelä 2775 & $3.9-4.8(-4.9) \times(2.7-) 2.8-3.1(-3.3)$ & 4.22 & 2.94 & $1.30-1.57$ & 1.43 \\
\hline Niemelä 4011 & $(4.0-) 4.1-4.9(-5.1) \times(2.6-) 2.7-3.1$ & 4.51 & 2.90 & $1.28-1.75$ & 1.55 \\
\hline Niemelä 5904 & $(3.8-) 4.0-4.9(-5.0) \times(2.4-) 2.5-3.0(-3.1)$ & 4.43 & 2.87 & $1.33-2.00$ & 1.54 \\
\hline 1973 Niemelä, Kotl. \& Pouz. & $(4.0-) 4.1-4.7(-5.0) \times 2.3-2.9$ & 4.41 & 2.68 & $1.45-1.96$ & 1.64 \\
\hline Penttilä $1245 b$ & $(3.5-) 3.7-4.4(-4.8) \times(2.4-) 2.6-3.0$ & 4.00 & 2.78 & $1.28-1.58$ & 1.44 \\
\hline Penttilä 1646 & $(4.0-) 4.1-4.8(-4.9) \times 2.8-3.1$ & 4.42 & 2.94 & $1.33-1.71$ & 1.51 \\
\hline Penttilä $1772 b$ & $(4.1-) 4.2-5.5(-6.2) \times(2.8-) 3.0-3.3(-3.5)$ & 4.97 & 3.10 & $1.37-1.88$ & 1.60 \\
\hline 1990 Pieri & $(4.0-) 4.1-5.2(-5.9) \times 2.4-3.0(-3.2)$ & 4.65 & 2.80 & $1.43-2.07$ & 1.66 \\
\hline 1930 Pilát (P. subpudorina) & & 4.98 & 3.07 & & 1.62 \\
\hline Saarenoksa 11487 & $4.0-5.0(-5.1) \times 2.5-3.0(-3.1)$ & 4.65 & 2.82 & $1.40-1.81$ & 1.65 \\
\hline Saarenoksa 34391 & $4.3-5.4(-5.5) \times(2.5-) 2.6-3.0$ & 4.98 & 2.83 & $1.43-2.08$ & 1.76 \\
\hline Saarenoksa 04496 & $(3.4-) 3.8-4.6(-5.0) \times(2.4-) 2.6-3.0(-3.3)$ & 4.17 & 2.84 & $1.33-1.78$ & 1.47 \\
\hline Saarenoksa 10096 & $(4.3-) 4.6-6.4(-6.9) \times(2.5-) 2.8-3.1(-3.2)$ & 5.57 & 2.95 & $1.43-2.30$ & 1.89 \\
\hline 1966 Shukov & $(4.2-) 4.3-5.3(-5.8) \times(2.4-) 2.6-3.0(-3.1)$ & 4.80 & 2.83 & $1.47-2.00$ & 1.70 \\
\hline Vehmaa 852 & $(3.8-) 4.0-4.7(-5.2) \times(2.6-) 2.7-3.1(-3.2)$ & 4.21 & 2.89 & $1.25-1.69$ & 1.46 \\
\hline \multicolumn{6}{|l|}{ C. resinascens } \\
\hline 2003 Halonen et al. & $(4.5-) 4.9-6.1(-6.3) \times 2.3-2.7(-2.9)$ & 5.52 & 2.50 & $1.80-2.38$ & 2.21 \\
\hline 1942 Jakobsson & $(4.0-) 4.3-5.0(-5.3) \times(2.1-) 2.2-2.7(-2.8)$ & 4.63 & 2.41 & $1.65-2.36$ & 1.92 \\
\hline Kinnunen 1 & $(3.9-) 4.0-5.0(-5.4) \times 2.2-2.9(-3.2)$ & 4.60 & 2.52 & $1.55-2.23$ & 1.83 \\
\hline 1936 Laurila & $(5.0-) 5.3-6.2(-6.3) \times 2.2-2.8(-2.9)$ & 5.78 & 2.48 & $2.08-2.70$ & 2.33 \\
\hline Laurila 549 & $4.2-5.0(-5.2) \times(2.2-) 2.3-2.8(-2.9)$ & 4.54 & 2.56 & $1.50-2.09$ & 1.78 \\
\hline Niemelä 532 & $(4.2-) 4.6-5.5(-6.2) \times(2.2-) 2.3-2.6$ & 5.09 & 2.43 & $1.68-2.38$ & 2.10 \\
\hline Niemelä 2051 & $(4.5-) 4.7-5.5(-6.2) \times(2.2-) 2.3-2.7(-2.8)$ & 5.07 & 2.45 & $1.67-2.48$ & 2.07 \\
\hline Niemelä 3281 & $4.1-5.1(-5.2) \times 2.2-2.7(-2.8)$ & 4.56 & 2.48 & $1.57-2.14$ & 1.84 \\
\hline Niemelä 6823 & $(4.3-) 4.5-5.2(-5.3) \times 2.3-2.8(-2.9)$ & 4.90 & 2.58 & $1.48-2.26$ & 1.90 \\
\hline 1951 Parmasto (T. polyetes) & $(4.4-) 4.6-5.2(-5.5) \times 2.1-2.4(-2.5)$ & 4.92 & 2.27 & $1.91-2.52$ & 2.17 \\
\hline 1991 I. Parmasto & $(4.2-) 4.4-5.2(-5.4) \times 1.9-2.1(-2.2)$ & 4.78 & 2.03 & $1.91-2.70$ & 2.36 \\
\hline Penttilä 4453 & $(4.2-) 4.4-5.2 \quad \times 2.3-2.9$ & 4.79 & 2.60 & $1.64-2.26$ & 1.84 \\
\hline Renvall 77 \& Renvall & $(4.0-) 4.3-5.3(-5.6) \times 2.3-2.8(-2.9)$ & 4.87 & 2.56 & $1.43-2.30$ & 1.90 \\
\hline Renvall 986 \& Renvall & $(4.0-) 4.2-5.0(-5.3) \times(2.2-) 2.3-2.6(-2.8)$ & 4.51 & 2.40 & $1.59-2.17$ & 1.88 \\
\hline Renvall 1387 \& Renvall & $(4.6-) 4.8-6.2(-6.3) \times 2.2-2.7(-2.8)$ & 5.41 & 2.44 & $1.77-2.70$ & 2.22 \\
\hline Romell 43 (type) & $(5.1-) 5.2-6.1(-7.5) \times(2.2-) 2.3-2.8(-3.1)$ & 5.77 & 2.61 & $1.96-2.42$ & 2.21 \\
\hline Saarenoksa 23784 & $(4.8-) 5.0-6.4(-6.5) \times(2.1-) 2.2-2.5$ & 5.56 & 2.35 & $2.09-2.71$ & 2.37 \\
\hline Saarenoksa 05185 & $4.3-5.6(-5.9) \times 2.3-2.6(-2.7)$ & 5.00 & 2.45 & $1.76-2.26$ & 2.04 \\
\hline
\end{tabular}


Appendix 1, contd.

\begin{tabular}{|c|c|c|c|c|c|}
\hline Specimen & $\mathrm{L} \times \mathrm{W}$ & $\mathrm{L}^{*}$ & $\mathrm{~W}^{*}$ & Q & $\mathrm{Q}^{*}$ \\
\hline Saarenoksa 11790 & $(3.8-) 4.0-4.7(-5.0) \times 2.3-2.7(-2.8)$ & 4.29 & 2.52 & $1.46-1.96$ & 1.70 \\
\hline Saarenoksa 28391 & $4.2-5.0(-5.1) \times 2.3-2.7(-2.8)$ & 4.65 & 2.45 & $1.56-2.17$ & 1.90 \\
\hline Saarenoksa 11292 & $(3.8-) 4.0-6.2(-6.5) \times(2.2-) 2.3-2.7(-2.8)$ & 5.06 & 2.45 & $1.65-2.70$ & 2.07 \\
\hline Saarenoksa 09894 & $(4.5-) 4.6-5.8(-6.1) \times 2.2-2.6(-2.7)$ & 5.22 & 2.36 & $1.92-2.55$ & 2.21 \\
\hline Söderholm 2122 & $(4.2-) 4.5-5.7(-6.1) \times(2.2-) 2.4-2.7(-2.8)$ & 5.06 & 2.49 & $1.72-2.55$ & 2.03 \\
\hline Vampola (Exsicc. 89) & $(4.5-) 4.6-5.8(-6.1) \times 2.2-2.6$ & 5.11 & 2.33 & $1.88-2.65$ & 2.19 \\
\hline \multicolumn{6}{|l|}{ C. balaenae } \\
\hline Haikonen 15315 & $(3.7-) 3.8-4.2 \quad \times(2.4-) 2.5-3.0(-3.2)$ & 4.01 & 2.78 & $1.28-1.67$ & 1.44 \\
\hline Heinonen $30895 \mathrm{~F}$ & $(3.4-) 3.6-4.1(-4.5) \times(2.4-) 2.6-3.0$ & 3.88 & 2.78 & $1.23-1.73$ & 1.40 \\
\hline 2.II.1991 Kamula & $(3.8-) 3.9-4.5(-5.0) \times(2.4-) 2.6-3.0(-3.2)$ & 4.16 & 2.77 & $1.27-1.71$ & 1.50 \\
\hline 15.II.1991 Kamula & $(3.7-) 4.0-4.6(-4.7) \times(2.4-) 2.6-3.0$ & 4.21 & 2.79 & $1.28-1.68$ & 1.51 \\
\hline Kotiranta 6371 & $(3.6-) 3.8-4.6(-4.9) \times(2.5-) 2.6-3.1(-3.3)$ & 4.11 & 2.90 & $1.27-1.70$ & 1.42 \\
\hline Niemelä 408b & $(3.5-) 3.7-4.1(-4.5) \times(2.4-) 2.6-3.0(-3.1)$ & 3.90 & 2.72 & $1.14-1.67$ & 1.43 \\
\hline Niemelä 2750 & $(3.4-) 3.6-4.2 \times(2.6-) 2.7-3.0(-3.1)$ & 3.85 & 2.87 & $1.23-1.50$ & 1.34 \\
\hline Niemelä 2752 (type) & $(3.6-) 3.8-4.3 \quad \times(2.5-) 2.6-3.0$ & 4.09 & 2.83 & $1.31-1.45$ & 1.45 \\
\hline Niemelä 2753 & $4.0-5.3(-5.6) \times(2.7-) 2.8-3.1(-3.6)$ & 4.52 & 2.98 & $1.33-1.71$ & 1.52 \\
\hline Saarenoksa 18894 & $(3.4-) 3.6-4.2(-4.3) \times(2.5-) 2.7-3.0(-3.1)$ & 3.91 & 2.86 & $1.26-1.44$ & 1.37 \\
\hline Saarenoksa 27794 & $(3.6-) 3.7-4.1(-4.4) \times(2.7-) 2.8-3.1(-3.3)$ & 3.93 & 2.96 & $1.21-1.40$ & 1.33 \\
\hline Saarenoksa 16395 & $3.6-4.3(-4.5) \times(2.7-) 2.8-3.2(-3.3)$ & 3.99 & 2.98 & $1.20-1.63$ & 1.34 \\
\hline Saarenoksa 16495 & $(3.2-) 3.3-4.1(-4.3) \times 2.5-3.0$ & 3.62 & 2.77 & $1.14-1.54$ & 1.31 \\
\hline Saarenoksa 08096 & $(3.6-) 3.8-4.2(-4.5) \times 2.7-3.2$ & 4.05 & 2.92 & $1.37-1.50$ & 1.39 \\
\hline 19.II.1994 Toresson & $(3.8-) 4.0-4.8(-5.0) \times 2.9-3.5(-3.8)$ & 4.26 & 3.15 & $1.24-1.44$ & 1.35 \\
\hline 4. XII.1994 Toresson & $(3.7-) 3.9-4.3 \quad \times 2.9-3.4$ & 4.06 & 3.13 & $1.18-1.43$ & 1.30 \\
\hline \multicolumn{6}{|l|}{ C. aneirina } \\
\hline 1915 Bondartsev & $(5.6-) 6.0-7.0(-7.2) \times(3.6-) 3.8-4.1(-4.2)$ & 6.44 & 3.92 & $1.44-1.89$ & 1.64 \\
\hline 1968 Bondartseva & $(5.0-) 5.3-6.6(-7.1) \times(3.1-) 3.3-4.0(-4.2)$ & 5.75 & 3.59 & $1.28-1.94$ & 1.60 \\
\hline Bonn 207 & $(4.6-) 5.3-7.0(-7.2) \times(3.4-) 3.6-4.1(-4.3)$ & 6.01 & 3.83 & $1.35-1.71$ & 1.57 \\
\hline Haikonen 6620 & $5.0-6.3(-6.7) \times(3.0-) 3.5-4.1(-4.2)$ & 5.61 & 3.85 & $1.28-1.63$ & 1.46 \\
\hline Yavorskiy 730 & $(5.2-) 5.3-7.2(-7.8) \times(3.5-) 3.6-4.2$ & 6.13 & 3.82 & $1.30-2.05$ & 1.60 \\
\hline Karsten 2505, 2506 (A. serena) & & 6.70 & 4.18 & & 1.60 \\
\hline Krawtzew W 182 (P. wasjug.) & & 6.93 & 4.05 & & 1.71 \\
\hline Laurila 715 & $(5.3-) 5.8-7.2(-8.1) \times(3.5-) 3.6-4.1(-4.3)$ & 6.53 & 3.83 & $1.23-2.03$ & 1.70 \\
\hline Niemelä 7249 & $(5.3-) 5.5-6.8(-6.9) \times 3.7-4.2$ & 6.06 & 3.98 & $1.39-1.68$ & 1.52 \\
\hline Niemelä 7250 & $(5.5-) 6.2-7.4(-7.5) \times(3.6-) 3.8-4.3(-4.5)$ & 6.52 & 4.05 & $1.45-1.76$ & 1.61 \\
\hline Saarenoksa 37086 & $(5.4-) 5.7-6.8(-6.9) \times 3.5-4.2(-4.3)$ & 6.16 & 3.86 & $1.42-1.83$ & 1.59 \\
\hline 1965 Shukov & $(6.1-) 6.7-8.5(-9.2) \times(3.0-) 3.2-3.8$ & 7.50 & 3.50 & $1.89-2.49$ & 2.14 \\
\hline 1820 Sommerfelt (type) & & 6.83 & 3.98 & & 1.72 \\
\hline \multicolumn{6}{|l|}{ C. cremea } \\
\hline Parmasto TAA 12481 & $(3.7-) 3.9-5.2(-5.9) \times(2.7-) 2.8-3.5(-3.6)$ & 4.51 & 3.02 & $1.32-1.71$ & 1.49 \\
\hline Parmasto TAA 13599 (type) & $(3.8-) 4.0-4.8(-5.0) \times(2.6-) 2.7-3.1(-3.2)$ & 4.31 & 2.88 & $1.31-1.72$ & 1.50 \\
\hline Parmasto TAA 55919 & $(4.2-) 4.6-6.4(-6.9) \times(2.7-) 2.9-3.5(-3.8)$ & 5.22 & 3.10 & $1.39-2.16$ & 1.68 \\
\hline Parmasto TAA 151113 & $3.9-5.2(-5.5) \times 2.7-3.1$ & 4.56 & 2.95 & $1.38-1.78$ & 1.55 \\
\hline \multicolumn{6}{|l|}{ C. albonigrecens } \\
\hline Parmasto TAA 52514 (type) & $(2.9-) 3.0-3.4(-3.7) \times(1.6-) 1.7-2.0$ & 3.19 & 1.79 & $1.61-2.00$ & 1.78 \\
\hline \multicolumn{6}{|l|}{ C. subrufa } \\
\hline 1990 Vampola (Exsicc.74) & $(4.8-) 5.0-6.0(-7.0) \times 3.6-4.2(-4.5)$ & 5.56 & 3.91 & $1.23-1.67$ & 1.42 \\
\hline
\end{tabular}

